\title{
The collateral pretraining of spaced responding ',
}

HANK DAVIS, UNIVERSITY OF MARYLAND

\author{
LADD WHEELER ${ }^{3}$, NAVAL MEDICAL RESEARCH INSTITUTE, BETHESDA, MARYLAND
}

Long Evans rats obtained a high frequency of reinforcement during initial exposure to a schedule which reinforced only responses spaced by at least $10 \mathrm{sec}$ (DRL 10) provided this schedule was part of a two lever concurrent Fixed RatioDRL 10 schedule on which (1) the animal had received Fixed Ratio pretraining and (2) the animal needed 10 or more sec to complete the ratio requirement. Subsequent removal of the ratio lever resulted in marked disruption of spaced responding, although removal of Fixed Ratio reinforcement did not affect spaced responding.

On the DRL schedule, responses are reinforced only if they are spaced by a specified interresponse time (IRT). In one of the earlier systematic analyses of DRL behavior, Wilson \& Keller (1953) noted the existence of "collateral responses, " repeatedly emitted sequences of behavior not directly reinforced or specified by the schedule contingency. In an experimental analysis of this behavior, Hodos, Ross, \& Brady stated that "... sequences of these collateral responses appear to mediate, at least in part, the timing process required for reinforced performance on the DRL schedule" (1962, p. 473). There has been relatively little study of these collateral responses which appear to be related to the species of the $S$, the cage topography, and the temporal requirements of the DRL. The research reported below is concerned with the effects of readily quantifiable pretrained collateral responses on DRL performance.

\section{Experiment 1}

Subjects. The Ss were three male Long Evans rats, two months old at the start of training. They were maintained at approximately $80 \%$ of their ad lib feeding weights.

Apparatus. An experimental chamber was sectioned into two compartments, each 12 in. $x 6$ in. $x 12$ in. (length $x$ width $x$ height), with a removable Plexiglas partition. Both sides of the cage were equipped with a lever, feeder, and food tray. Individual feeders automatically delivered $45 \mathrm{mg}$ Noyes pellets.

Training. Rats No. 5, No. 10, and No. 20 were trained to stable performance on Fixed Ratio (FR) 5, FR 10, and FR 20 schedules, respectively. They were given training sessions consisting of 100 reinforcements, five days a week for six weeks. During training, the animals were restricted by the Plexiglas divider to the left half of the cage.

Test session. During the test session, the cage divider was removed, allowing the animal access to his training lever (programmed to the training schedule) and to the right lever which was programmed on a DRL 10. The schedules were operated concurrently.

Results. All animals rapidly developed switching be- havior between the two levers, i.e., following reinforcement on one lever, the animal began to work on the second lever. Figure 1 presents the cumulative record of each animal's DRL responses. Whereas Ss No.10 and No. 20 made only 70 and 47 unreinforced DRL responses, respectively (responses occurring sooner than the specified 10 sec IRT), S No. 5 emitted 493 such responses during the test session.

Discussion. The initial high frequency of reinforcement attained by Ss No.10 and No. 20 on the DRL schedule appeared to be related not only to the availability of the FR lever, but also to the specific size of the FR schedule on which they were run. That is, because the length of time taken to complete the FR requirement largely determined the inter-response times on the DRL schedule, Ss No. 10 and No. 20 received a high frequency of DRL reinforcement because responding on their FR schedules consistently exceeded the $10 \mathrm{sec}$ requirement for DRL reinforcement. However, S No. 5's switching behavior did not result in DRL reinforcement since the time taken to emit the five responses for FR reinforcement did not reliably produce a sufficient interresponse time for DRL reinforcement.

\section{Experiment 2}

The previous experiment demonstrated that switching behavior emerging on initial exposure to a concurrent FR DRL schedule will result in a high frequency of DRL reinforcement, provided that the FR requirement produces behavior which temporally exceeds the DRL requirement. Experiment II was directed at demonstrating that the reinforced DRL behavior obtained during the

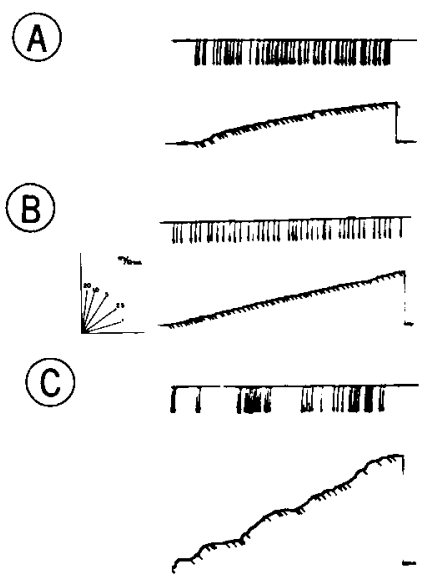

Fig. 1. Cumulative DRL response curves obtained during initial exposure to concurrent Fixed Ratio DRL 10 schedules for Rats (A) No. 10, (B) No. 20, and (C) No. 5 following collateral pretraining on Fixed Ratio 10, FR 20, and FR 5 respectively. Event marker above record signifies Fixed Ratio reinforcement. Blips on response record indicate DRL reinforcements. 

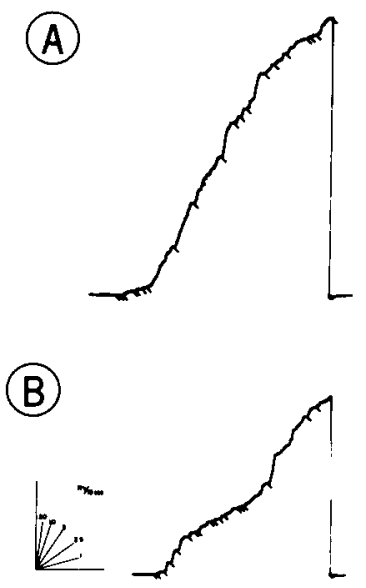

Fig. 2. Cumulative DRL response curves for (A) Rat No. 10 and (B) Rat No. 20 following insertion of the cage divider restricting the animal to the DRL lever.

initial session was indeed dependent on the availability of the FR lever.

Subjects. Rats No. 10 and No. 20.

Test session. On the day following the test session of Experiment I, the divider was inserted into the cage, restricting the animal to the DRL lever for a period of 20 reinforcements. The session was then terminated.

Results. Figure 2 presents the cumulative records of responses emitted on the DRL lever by Ss No. 10 and No. 20 following insertion of the Plexiglas divider into the cage. Ss No. 10 and No. 20 emitted 407 and 342 unreinforced DRL responses respectively during the period in which each received only 20 reinforcements on the DRL schedule.

Discussior. It is apparent that the high frequency of DRL reinforcement obtained by these Ss in Experiment I was specifically related to the availability of the FR schedule on which they had been pretrained; removal of the FR lever resulted in a low frequency of DRL reinforcement.

\section{Experiment 3}

In Experiment I, Rats No. 10 and No. 20 emitted their reinforced DRL responses in the pause times following Fixed Ratio reinforcement. The purpose of this experiment was to investigate whether an animal would continue to respond on both levers if reinforcement were withheld on the FR schedule.

Subjects. Rat No. 10.

Training. The animal was run on a FR 10 schedule for 10 additional sessions of 100 reinforcements each, during which he was restricted to the left side of the cage as during original training.

Test sessions. The animal was run for 12 sessions, during which he had access to both levers. The left lever (previously programmed on FR 10) was no longer programmed to deliver reinforcement (extinction), while the right lever was maintained on a DRL 10 schedule. Each session was terminated after $30 \mathrm{~min}$.
Results. No systematic decrease appeared in the number of responses emitted on the FR lever in the absence of primary reinforcement on this schedule $(\bar{X}=731 /$ session; $\mathrm{SD}=76.4$; range $=834$ (Session 8$)-625$ (Session 5$)$. Moreover, the S continued his lever switching behavior throughout all sessions and responded on the DRL lever with sufficient interresponse times to maintain a high density of reinforcement $(\overline{\mathrm{X}}=105.8$ /session; $\mathrm{SD}=6.3$; range $=92$ (Session 2$)-130$ (Session 7$)$.

Discussion. The maintenance of spaced responding under these conditions may be viewed as performance on an analogous two lever chained schedule (chain FR 10 CRF) (Ferster \& Skinner, 1957). That is, the animal's behavior in the concurrent FR ext DRL 10 situation is that of a chained contingency of 10 left lever presses and one right lever press for reinforcement.

\section{Conclusion}

It is concluded from this series of experiments that collateral pretraining on a temporally sufficient Fixed Ratio schedule will result in good initial DRL performance in the concurrent FR DRL situation. Moreover, such DRL performance may be maintained in the absence of FR reinforcement.

Although the spaced responding given in these experiments may be viewed as being artifactual or highly dependent on specific schedule arrangements, its appearance is almost immediate and the $E$ retains the option of discontinuing reinforcement of the pretrained behavior without disrupting the animal's spaced responding. During traditional DRL training, much time is often spent in waiting for the animal to emit and stabilize on a collateral sequence which will span the interresponse time requirement of the schedule. Where rapidly attained spaced responding is required, it may therefore be expedient for the $\mathrm{E}$, rather than the $\mathrm{S}$, to select the collateral behavior and to condition it as he would any other operant response.

\section{References}

Ferster, C. B., \& Skinner, B. F. Schedules of reinforcement. New York: Appleton-Century-Crofts, 1957.

Hodos, W., Ross, G. C., \& Brady, J. V. Complex response patterns during temporally spaced responding. J. exp. Anal. Behav., 1962, 4, 473-479.

Wilson, M. P., \& Keller, F. S. On the selective reinforcement of spaced responses. J. comp. physiol. Psychol., 1953, 46, 190-193.

\section{Notes}

1. From Bureau of Medicine and Surgery, Navy Department, Research Task MFO22.01.03-1002. The opinions and statements contained herein are the private ones of the writers and are not to be construed as official or as reflecting the views of the Navy Department or the Naval Service at large.

2. The experiments reported herein were conducted according to the principles enunciated in "Guide for Laboratory Animal Facilities and Care" prepared by the Committee on the Guide for Laboratory Animal Resources, National Academy of Sciences-National Research Council.

3. Our thanks to Evelyn E. Lett for running SS in Experiment III. Our thanks also to T. Daryl Hawkins and Mary E. Wheeler for critical reviews of an earlier draft of this manuscript. 